\title{
Generating Full Paraphrases of Fragments in a Dialogue Interpretation System
}

\author{
Christian Ebert \\ Shalom Lappin \\ Howard Gregory \\ Department of Computer Science \\ King's College London \\ \{ebert, lappin\}@dcs.kcl.ac.uk \\ howard.gregory@kcl.ac.uk
}

\author{
Nicolas Nicolov \\ IBM T. J. Watson Research Center \\ Yorktown, New York \\ nicolas@watson . ibm.com
}

\begin{abstract}
Using SHARDS - a semanticallybased HPSG approach to the resolution of dialogue fragments - we will show how to generate full paraphrases for fragments in dialogue. We adopt a template-filler approach that does not require deep generation from an underlying semantic representation. Instead it reuses the results of the parse and interpretation process to dynamically compute templates and to update fillers as the dialogue proceeds. This recycling of already available syntactic and phonological information makes generation efficient, as it reduces the operations of the generator to mere string manipulations.
\end{abstract}

\section{Introduction}

Much previous work on generation has focused on the general problem of producing lexical strings from abstract semantic representations. We consider generation in the context of a particular task, creating full sentential paraphrases of fragments in dialogue. When the syntactic, semantic and phonological information provided by a dialogue fragment resolution system is made accessible to a generation component, much of the indeterminacy of lexical selection is eliminated.

One major challenge for any dialogue interpretation system is the proper treatment of fragments. Examples include bare NP answers (2a), where the NP a personnel manager is resolved as the assertion (2b), and sluicing (4a), where the $w h$-phrase is interpreted as the question $(4 \mathrm{~b})^{1}$.

(1) Who hired Jones?

(2) (a) A personnel manager.

(b) A personnel manager hired Jones.

(3) A personnel manager hired Jones.

(4) (a) Who?

(b) Which personnel manager hired Jones?

Furthermore the antecedent or the fragment may be embedded, as in the following examples.

(5) The management asked who hired Jones.

(6) (a) The personnel department thinks a board member.

(b) The personnel department thinks a board member hired Jones.

(7) The personnel department thinks a board member hired Jones.

(8) (a) The CEO wonders who.

(b) The CEO wonders which board member hired Jones.

\footnotetext{
${ }^{1}$ Throughout this paper sentences representing paraphrases will be set in italics.
} 
Generating full paraphrases for interpreted fragments in a dialogue management system is an important utility for facilitating humanmachine communication. We consider three cases where paraphrase generation performs a useful function.

Dialogue Systems In dialogue systems the purpose of paraphrase generation is twofold. First, it increases the explicitness and transparency of the system, which might be an option that the user wishes to use. Second, it is necessary for the generation of clarification questions, which can be used by the system to resolve ambiguity. This is illustrated in the following dialogue between a user and an information system.

USER: Who did the personnel department hire?

SYSTEM: John thinks a student.

USER: Who?

At this point Who? might ask for more details about John (because the user wonders who John is) or it might be a question about the hired student. To resolve this ambiguity the system produces the following clarification question by generating the paraphrase of the user's wh-sluice:

\section{System: Do you want to know which stu- dent John thinks the personnel department hired?}

If the user answers positively the system responds with the fragment answer or the full paraphrase, depending on the parameter which controls the system's level of explicitness:

System: Bill Smith.

or

System: John thinks the personnel department hired Bill Smith.

If the user answers negatively, then the system will treat Who? as a clarificatory question on $J o h n^{2}$.

System Evaluation A natural application of paraphrase generation is the monitoring of a system's performance. Full paraphrases can be used to interactively test the system's interpretation of fragments. When the paraphrase is available to a human user, he/she can confirm or revise the paraphrase, and so monitor the performance of the system efficiently. The paraphrase generator that we present in this paper is currently used for monitoring the performance of the SHARDS system - a system for resolving fragments in dialogue - which is introduced in more detail in section 2 .

Machine Translation Certain elided structures pose a problem for machine translation. Although the source language might exhibit ellipsis structures of a specific kind, the target language might not allow for these. Therefore these structures will have no direct translation. Two cases in point are VP-ellipsis and pseudo-gapping in English, which have no direct counterparts in languages such as German. A straightforward solution is to use full paraphrases instead of ellipsis as the input to the MT component. Consider the following English dialogue and it's translation into German ${ }^{3}$.

(9) (E) Who submitted a report today?

(G) Wer legte heute einen Bericht vor?

(10) (E) John did to his supervisor.

(G) John ?[*tat es] seinem Betreuer.

(11) (E) John submitted a report to his supervisor today.

(G) John legte heute einen Bericht seinem Betreuer vor.

\footnotetext{
${ }^{2}$ See (Ginzburg, 2001) for an account of clarificatory ellipsis within the general HPSG framework assumed here. A procedure for recognizing clarificatory question fragments is being developed on the basis of this account.

${ }^{3}$ Currently our system is not capable of handling ellipsis structures. But as we point out in the last section, we are extending it to deal with these cases.
} 
The English answer (10E) exhibits pseudogapping, which cannot be translated into a similar structure in German (10G). A translation including an auxiliary corresponding to did is ungrammatical. Dropping the auxiliary prior to the translation results in an (at best) odd sentence.

This problem can be circumvented using paraphrase generation. Instead of translating (10E) directly, it's paraphrase (11E) is computed. Then the translation can proceed with this paraphrase as the source input to obtain an appropriate German sentence (11G).

Using the fragment interpretation system SHARDS we show how to generate paraphrases for fragments in dialogues like those in (b) in the examples (1-8) above. The generator uses a template-filler approach, and it does not do any deep generation from an underlying semantic representation. Instead it reuses the results of the parse and interpretation process of SHARDS to dynamically compute the templates, and then to update the filler. This recycling of already available syntactic structures makes generation efficient because it reduces the operations of the generator to string manipulations.

In Section 2 we give a brief review of the SHARDS system. We then explain our proposal for generating fragment paraphrases with templates in Section 3. In Section 4 we briefly describe the implementation of SHARDS and the generation component. Section 5 sketches some directions for future work.

\section{The SHARDS System}

SHARDS (Ginzburg et al., 2001) is a Head Driven Phrase Structure Grammar (HPSG)based system for the resolution of fragments in dialogue. It is based on a version of HPSG developed in (Ginzburg and Sag, 2000) which integrates the situation semantics-based theory of dialogue context given in the KOS framework (Cooper et al., 1999) into recent work in HPSG (Pollard and Sag, 1994; Sag, 1997). Following (Ginzburg and Sag, 2000), two new attributes are defined within the CONTEXT feature structure: the Maximal
Question Under Discussion (MAX-QUD) and the Salient Utterance (SAL-UTT).

The MAX-QUD ${ }^{4}$ can be seen as the most salient question that needs to be answered in the course of a dialogue. It's value is of type question. In the framework of this system, questions are represented as semantic objects comprising a set of parameters - that is, restricted indices - and a State of Affairs (SOA) (12).

$$
\left[\begin{array}{ll}
\text { question } & \\
\text { PARAMS } & \{\pi, \ldots\} \\
\text { SOA } & {[\operatorname{soa}(\ldots \pi \ldots)}
\end{array}\right]
$$

This is the feature structure counterpart of the $\lambda$-abstract $\lambda \pi .(\ldots \pi \ldots)$. In a $w h$-question the PARAMS set represents the abstracted INDEX values associated with the $w h$-phrase(s). For a polar (yes-no) question the PARAMS set is empty. In general a number of such questions may be available in a given dialogue context, of which one is selected as the value of MAX-QUD.

The SAL-UTT represents a distinguished constituent of the utterance whose content is the current value of MAX-QUD. In information structure terms, it can be thought of as specifying a potential parallel element correlated with an element in the antecedent question or assertion. It's value is of type sign, enabling the system to encode syntactic categorial parallelism, including case assignment for the fragment.

Specifically, SAL-UTT is computed as the (sub)utterance associated with the role bearing widest scope within MAX-QUD:

- For $w h$-questions, SAL-UTT is the $w h$ phrase associated with the PARAMS set of the question.

- If MAX-QUD is a question with an empty PARAMS set, the context will be underspecified for SAL-UTT. The possible values for the SAL-UTT feature are either

\footnotetext{
${ }^{4}$ In the following we will use 'MAX-QUD' as an abbreviation for 'maximal question under discussion' as well as for the corresponding HPSG feature
} 
the empty set or the utterance associated with the widest scoping quantifier in MAX-QUD. This is envoked to resolve sluicing ${ }^{5}$. In the case of polar questions, SAL-UTT will be empty.

Interpreting a bare phrase $B$ in dialogue consists in computing from context (represented as a dialogue record) the MAX-QUD and SAL-UTT features of the assertion or question clause $B C L$ that $B$ expresses, and then using these features to specify the CONTENT feature of $B C L$.

Bare argument phrases constitute a nonhead daughter (the fragment), the remaining information for the interpretation being provided primarily by the contextual features. Phrases are classified not only in terms of their phrasal type, but also with respect to the further dimension of clausality. Fragments are assigned to a subtype of the type bare-arg-ph, namely to bare-decl-cl for 'short answers' or bare-wh-cl for sluices.

The restrictions on interpretation are then imposed by constraints on these types. The constraint on bare-arg-ph, for instance, requires unification of the NUCLEUS (propositional core) feature of the MAX-QUD and the CONTENT feature of the proposition or question of which the bare argument phrase is the non-head daughter. Similarly, the CAT feature of the SAL-UTT and the non-head daughter features of the bare phrase are unified to insure syntactic categorial parallelism. The constraint on bare-decl-cl requires the RESTR value of the phrase to be the union of the RESTR values of the MAX-QUD and the non-head daughter (the fragment), i.e. it enforces the amalgamation of semantic restrictions given by the context and the fragment itself. For further details on the grammatical framework see (Sag, 1997; Ginzburg and Sag, 2000).

We give the result of resolving fragment (2a). After parsing the antecedent (1) and the fragment phrase, the resolution procedure yields the Attribute-Value-Matrix (AVM) in

\footnotetext{
${ }^{5}$ SAL-UTT can also be a set containing more than one element in contexts where MAX-QUD is a multiple question as in A: Who arrived when? B: Jo at 5, Mustafa at \%.
}

figure 1. The AVM satisfies the constraints mentioned above, as the NUCLEUS value of the entire clause for which the bare phrase is a non-head daughter is structure shared with MAX-QUD's NUCLEUS via 1 , categorial parallelism is ensured via 6 , and the semantic restrictions 2 and 3 have been amalgamated into the RESTR value of the phrase.

The generator uses AVMs like this to set up it's templates and fillers.

\section{Generation of Fragment Paraphrases}

Template-based approaches to NL generation have proved useful in various systems (see e.g. (Reiter, 1995; Becker and Busemann, 1999)). These approaches are particularly appropriate in systems where large parts of the text to be generated remain fixed in some way, or are partially determined prior to generation. An inspection of the following dialogue indicates that this is true for the generation of fragment paraphrases:

(13) The personnel department wonders who the CEO hired.

(14) (a) John thinks a student.

(b) John thinks the CEO hired a student.

(15) (a) Who?

(b) Which student did the CEO hire?

(16) (a) The student who passed all tests.

(b) The CEO hired the student who passed all tests.

The paraphrase $(14 \mathrm{~b})$ is a combination of parts of the fragment answer (14a) and the antecedent clause (13). It is worth noting at this point that dialogues with consecutive fragment answers or questions (such as the one above) are recursive in nature. The paraphrase (16b) for instance contains material from the initial clause of the discourse. Just considering the antecedent Who? and the fragment the student who passed all tests itself is not sufficient to generate the fragment's paraphrase. It is necessary to insure 


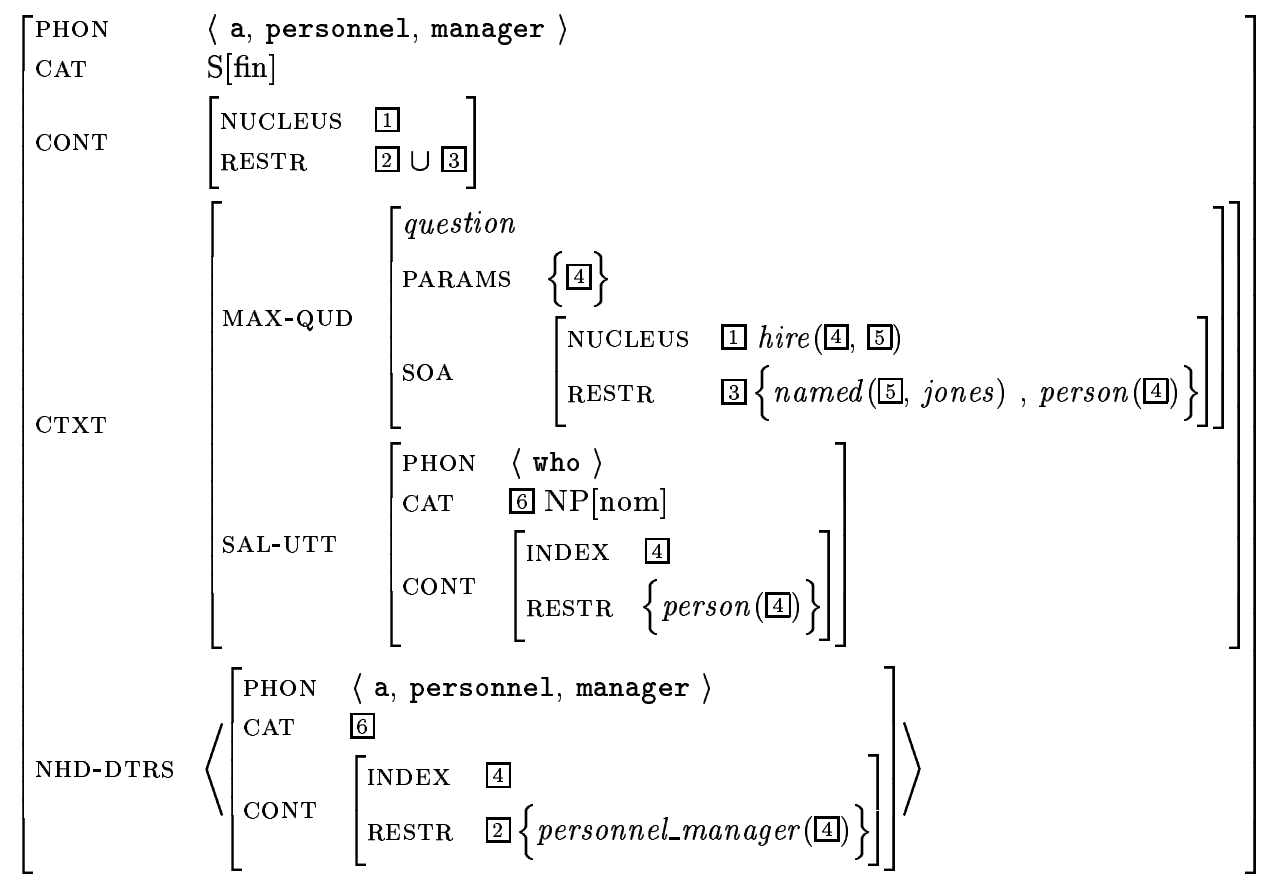

Figure 1: Resolution of (2a): A personnel manager hired Jones.

that some parts of the dialogue (in this case the CEO hired ...) are carried over from one paraphrase to the other, while other parts have to be modified with respect to the fragment (such as which student and the student who passed all tests). This suggests the use of templates for the fixed parts that have to be carried over, and of fillers for the parts that might require modification within the templates.

Unlike template-based generation systems where the templates are defined in advance, our algorithm uses dynamically created templates. The templates are not fixed in advance, but must be constructed dynamically from the dialogue. In a dialogue such as (1316) the templates are built once for a specified MAX-QUD at the very beginning and remain specified until the dialogue ends. More precisely, every change in the MAX-QUD forces a recomputation of the templates. Another dynamic feature concerns the filler. After each utterance, the filler is updated and combined with one of the templates to yield the intended paraphrase. This enables us to update the paraphrases in a sequence of questions and fragment answers.
The main point in using this template-filler mechanism is that it permits the reuse of constituents/syntactic structure that is already available. Since the surface strings are accessible from the user input, and the syntactic and semantic information is available from the parse and fragment interpretation, it is useful to exploit these resources. Our generator performs shallow generation by recycling already given data without using deep semantic knowledge.

We now consider the dynamic construction of the templates and fillers in greater detail. Essentially, the templates correspond to different paraphrase schema of the MAX-QUD, while the fillers correspond to the fragments.

\subsection{Identifying the Templates}

In (13-16) the parser identifies the question Who did the CEO hire? as the maximal question under discussion and who as the salient utterance. We can see that a certain constituent of the MAX-QUD - namely did the CEO hire - reappears in modified forms in the paraphrases $(14-16)(\mathrm{b})$ : The paraphrases (14b) and (16b) contain this component in Subject-Verb-Object (SVO) order (the CEO 
hired ...). The paraphrase (15b) however contains this component after Subject-AuxiliaryInversion (SAI) has been done (...did the CEO hire). To handle these phenomena our algorithm constructs two different templates: the template $T_{s v o}$ for the SVO case and $T_{s a i}$ for the SAI case.

Both templates contain a slot (represented by $\diamond$ ) which determines the position that the filler will occupy. In the example above we indicated this position with three points. For the dialogue in (13-16) the templates are

$$
\begin{aligned}
& T_{s v o}=\text { the CEO hired } \diamond \\
& T_{s a i}=\diamond \text { did the CEO hire }
\end{aligned}
$$

\subsection{Identifying the Filler}

The dialogue contains the following progression from the SAL-UTT who in (13) to the final bare NP answer the student who passed all tests in (16):

$$
\begin{gathered}
F_{1}: \text { who } \\
\downarrow \\
F_{2}: \text { a student } \\
\downarrow \\
F_{3}: \text { which student } \\
\downarrow \\
F_{4}: \text { the student who passed all tests }
\end{gathered}
$$

We generate the paraphrases of the corresponding fragments by substituting the fillers $F_{1}$ to $F_{4}$ for the slots in the corresponding templates. For instance substituting $F_{3}$ for the slot in $T_{s a i}$ (which we write $T_{s a i}\left(F_{3}\right)$ ) yields the intended paraphrase (15b).

Each fragment produces an update of the preceding filler. E.g. the filler $F_{2}$ is updated by the fragment who of (15a) to $F_{3}$. The final selection of the template and it's composition with the filler depends on the entire fragment clause and not on the fragment alone. In (14), for instance, we have to prefix John thinks to $T_{s v o}\left(F_{2}\right)$ to get the intended result.

\section{An Implemented System for Fragment Resolution and Paraphrase Generation}

The entire system of fragment resolution and generation is implemented in Prolog using
ProFIT (Erbach, 1996) as a formalism to handle typed feature structures. The complete system consists of five main components. The components I-IV constitute the SHARDS system (Ginzburg et al., 2001). V is the paraphrase generator.

I. HPSG Grammar. This is a substantially modified version of the grammar employed by (Gregory and Lappin, 1999), but using the types and features mentioned in Section 2.

II. Dialogue Record. When a clause has been parsed (and any fragments resolved), its AVM is first converted into a transitive network of Mother-DaughterRelations (the MDR list) and then stored in a dialogue record paired with an index (counter). A list of MAX-QUD candidates is computed from the CONT of each subclause and stored as a further component of the dialogue record.

\section{Context Resolution Procedure.}

This assigns values from the dialogue record to the MAX-QUD and SAL-UTT features of the current clause. The most recent element of the MAX-QUD candidate list which is compatible with the constraints imposed by the bare argument phrase is selected as the value of the MAX-QUD ${ }^{6}$. SAL-UTT is obtained from the sign whose content provides MAX-QUD by applying the conditions on scoped elements proposed in (Ginzburg et al., 2001).

\section{Fragment Resolution Procedure.}

This computes the semantics (i.e. the CONT) of the current fragment clause from its MAX-QUD and SAL-UTT values.

V. Paraphrase Generator. After the resolution procedure has been applied, the generator is called with the antecedent clause in the form of the MDR list and the resolved fragment clause in the form

${ }^{6}$ This recency-based procedure for selecting the MAX-QUD from the candidate list may require modification in light of future testing and evaluation 
of an AVM. It computes the templates, updates the fillers, and returns the paraphrase of the fragment clause.

\subsection{Implementation of the Generation Algorithm}

The generator takes AVMs as arguments (a list of AVMs as the Mother- DaughterRelations list corresponding to the antecedent and the AVM of the resolved fragment clause) and returns the paraphrase of the fragment clause in the form of a word string. The MDR list argument enables the generator to search the parse of the antecedent clause efficiently, e.g. for the auxiliary in SAI clauses or for some verbal head, etc. The operations (such as deletion or substitution of an element) that the generator performs are carried out on the surface strings, i.e. on the values of the PHON features. The generator extracts the PHON values of the AVMs for the antecedent clause and the fragment clause in the initial phase of generation and performs it's operations not on their AVMs but just on these surface strings. All operations - though guided by the information in the full AVMs are simple string manipulations.

If the generator is called for the first time (or when the MAX-QUD changes, e.g. when a new dialogue starts), it computes the two templates and the first filler $F_{1}$, using the antecedent clause and the fragment, according to the following algorithm:

\section{Construct Templates}

1. Identify the SAL-UTT and store the AVM as first filler $F_{1}$.

2. Identify the MAX-QUD and delete the SAL-UTT to get the AVM $M Q$.

3. Compute the templates $T_{s a i}$ and $T_{s v o}$ from $M Q$ and store them:

(a) If $M Q$ is in SVO order, store it as $T_{\text {svo }}$, compute $T_{s a i}$, and insert the slots; else

(b) $M Q$ is in SAI order, store it as $T_{s a i}$, compute $T_{s v o}$, and insert the slots.
The setup of the templates requires a conversion from a clause in SVO order to one in SAI order or vice versa. If the clause is in SVO order, the verbal head has to be searched, an appropriate auxiliary has to be inserted into the string, and the base form of the verb has to be substituted for it's inflected form. If the conversion has to be done in the other direction, then the auxiliary is identified and deleted, and the inflected form of the verb is substituted for it's base form.

The insertion of the slot at the appropriate position in the SVO template requires the identification of the verbal head which subcategorizes for the SAL-UTT. After this verb has been found, the slot is inserted at the position that the SAL-UTT would occupy. E.g. if the SAL-UTT is the subject, then the slot is inserted immediately in front of this verb. In the case of the SAI template the slot is inserted at the beginning of the template just in front of the auxiliary.

As mentioned above, the search for daughters such as the verbal heads can be done efficiently by going through the MDR list, which has already been computed for the SHARDS dialogue record (cf. component II above).

Once the templates are set up, the generator updates the filler $F_{i}$ to $F_{i+1}$ according to the type of the fragment. Below we refer to the clause which contains the fragment as $F C$.

\section{Update Filler}

1. If the fragment of $F C$ is a wh-phrase, substitute which for the determiner in the filler $F_{i}$; else,

2. substitute the fragment for $F_{i}$.

To accomplish the first case of filler update the AVM corresponding to the stored filler $F_{i}$ is searched for it's determiner. Then which is substituted for this determiner. The second case is straightforward.

After the filler has been updated the generator is ready to compute the complete paraphrase $P_{i+1}$. Let $\circ$ stand for the concatenation of strings and $\varepsilon$ for the empty string. We write $T(F)$ for the result of substituting the 
filler $F$ for the slot $\diamond$ in the template $T$. Thus $T(\varepsilon)$ is the result of deleting the slot from $T$. The composition of template and filler depends on the clause $F C$, which contains the fragment.

\section{Composition of template and filler}

1. If $F_{i+1}$ is a $w h$-phrase

(a) If $F C$ consists just of the fragment, then $P_{i+1}=T_{s a i}\left(F_{i+1}\right)$; else,

(b) substitute $F_{i+1} \circ T_{\text {svo }}(\varepsilon)$ for the fragment in $F C$ to get $P_{i+1}$;

else,

2. substitute $T_{\text {svo }}\left(F_{i+1}\right)$ for the fragment in $F C$ to get $P_{i+1}$.

The if-then cascade takes care of the possible word orders and specifically of the fronting of wh-phrases. 1(a) handles fronted wh-phrases in SAI constructions of wh-questions such as Which student did the CEO hire?. Case 1(b) handles cases where the wh-question is embedded, as in The personnel department wonders which student the CEO hired. Case 2 generates the SVO order in embedded and non-embedded cases ([John thinks] the CEO hired a student.).

Once the filler and the template have been set up, the composition of these two components is achieved by simple string concatenation. We insure agreement of the filler and the template by supplying the slot with the corresponding agreement features of the template and checking them when the filler is inserted ${ }^{7}$.

\subsection{The Generator at work}

We can now illustrate the generation algorithm with dialogue (13-16). The first time the generator is called the arguments are a parse (in the form of the MDR list) of the

\footnotetext{
${ }^{7}$ It will be necessary to refine our agreement checking procedure to deal with mismatches in number and tense, as in (i) and (ii) respectively.
}

(i) (a) Who is presenting the report?

(b) John and Mary.

(ii) (a) Who has written the program?

(b) Mary will. antecedent clause (13) and an AVM of the parsed and interpreted fragment clause (14). As this is the first call, the templates $T_{s v o}$ and $T_{s a i}$ are generated in the way described above. The first filler $F_{1}$ is set to the salient utterance who. The situation is as follows:

$$
\begin{aligned}
F_{1} & =\text { who } \\
T_{\text {svo }} & =\text { the CEO hired } \diamond \\
T_{\text {sai }} & =\diamond \text { did the CEO hire }
\end{aligned}
$$

Immediately after this initial computation the filler is updated to

$$
F_{2}=\text { a student }
$$

because the fragment is a student. As the filler is not a wh-phrase, case 2 of the composition step applies and $T_{s v o}\left(F_{2}\right)=$ the CEO hired a student is substituted for a student in (14). The final paraphrase is

$$
\begin{array}{r}
P_{2}=\text { John thinks the CEO hired } \\
\text { a student }
\end{array}
$$

The next call of the generator with (14) as the antecedent clause and (15) as the fragment clause leads to an update of $F_{2}$ to

$$
F_{3}=\text { which student }
$$

as the fragment is who and which is substituted for the determiner a in $F_{2}$. The composition yields

$$
P_{3}=\text { Which student did the CEO hire? }
$$

As $F_{3}$ is a $w h$-phrase and the fragment clause consists of the fragment who, case 1(a) is considered. If the bare phrase had been embedded, as in Bob wonders who, case 1(b) would lead to the substitution of $F_{3} \circ T_{s v o}(\varepsilon)$ for who, and the paraphrase would be Bob wonders which student the CEO hired. After the last call of the generator, the filler $F_{3}$ is updated to

$$
F_{4}=\text { the student who passed all tests }
$$

and the paraphrase is computed as

$$
\begin{array}{r}
P_{4}=\text { The CEO hired the student } \\
\text { who passed all tests }
\end{array}
$$


This algorithm works properly with examples that contain multiple embeddings, as in the case of the following antecedent clause, where the $w h$-phrase occurs outside of the matrix clause the system administrator thinks....

(17) Peter wonders who the system administrator thinks deleted the files.

(18) (a) The webmaster believes a student.

(b) The webmaster believes the system administrator thinks a student deleted the files.

(19) (a) Who?

(b) Which student does the system administrator think deleted the files?

The parser identifies the question Who does the system administrator think deleted the files as the maximal question under discussion and who as the salient utterance (and the first filler $F_{1}$ ). Therefore the antecedent clause (17) gives rise to the two templates

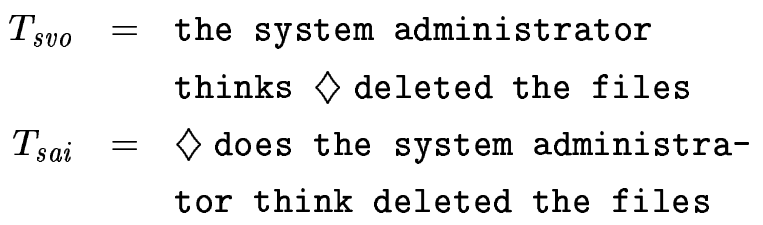

The first update of the filler yields $F_{2}=$ a student, and the paraphrase (18b) is generated on case 2 of the composition procedure. In the next turn $F_{2}$ is updated to $F_{3}=$ which student, and case $1(\mathrm{a})$ of the composition procedure leads to (19b).

The algorithm is able to produce paraphrases of answers to polar questions quite straightforwardly.

(20) Does Peter think the CEO hired a student?

(21) (a) Yes.

(b) Peter thinks the CEO hired a student.

(c) No.

(d) Peter does not think the CEO hired a student.
As the polar question (20) is an SAI construction we can just run the procedure for setting up the templates on it. This will compute $T_{s v o}$ and since the SAL-UTT is empty in the case of polar questions, nothing will be deleted, and no slot will be inserted. The result will be the paraphrase (21b). A small addition to this procedure makes it possible to generate the paraphrases of negative answers such as (21c) as well.

The generator can produce paraphrases for all phenomena that the parser and the resolution procedure of SHARDS currently handle. These are bare NP fragments and bare $w h$ sluices, embedded fragments and polar questions of the kind illustrated in the preceding sections.

\section{Conclusion and Future Research}

Most work on NL generation such as (Shieber et al., 1990; Kay, 1996; McKeown, 1985; Nicolov and Mellish, 2000) has formulated the problem in abstract terms as the production of a lexical string to encode a semantic representation. We have situated generation within the context of dialogue interpretation, specifically fragment resolution. In doing so, we have been able to eliminate much of the indeterminacy which characterizes classical generation systems by exploiting the rich syntactic and phonological information produced in the course of dialogue interpretation.

While there are undoubtly generation problems to which this approach does not apply, the work described here does suggest the possibility of efficient generation through the exploitation of the results of dialogue interpretation in an important class of NL applications.

The research on the system we introduced in the preceding sections is part of a larger project of dialogue management at King's College, London. We are extending our system in several directions. We plan to incorporate the handling of ellipsis structures (VPellipsis and gapping) into the SHARDS system, and the generation component will be developed to deal with these constructions. In addition, we are currently doing corpus work with the British National Corpus, which will 
eventually result in a typology of fragment types. We will use the examples of ellipsis that we find in this corpus to evaluate and improve our generation system. We are also in the process of extending the lexicon to achieve broader coverage for our parser and fragment interpretation components.

\section{Acknowledgements}

We are grateful to two anonymous reviewers for helpful comments on an earlier draft of this paper. The dialogue project of which the work described here is a part is funded by grant number R00022269 of the Economic and Social Research Council of the United Kingdom. Some of the research presented in this paper was done in the summer of 2000 , when the second author was a Visiting Academic at the IBM T.J. Watson Research Center in Hawthorne, NY. During this time he worked with the fourth author on the design and implementation of an initial version of the generation algorithm.

\section{References}

Tilman Becker and Stephan Busemann, editors. 1999. May I Speak Freely? Between Templates and Free Choice in Natural Language Generation. Workshop at the 23rd German Annual Conference for Artificial Intelligence (KI'g9), Saarbrücken. DFKI.

R. Cooper, S. Larsson, M. Poesio, D. Traum, and C. Matheson. 1999. Coding instructional dialogue for information states. In Task Oriented Instructional Dialogue (TRINDI): Deliverable 1.1. University of Gothenburg, Gothenburg.

Gregor Erbach. 1996. ProFIT: Prolog with features, inheritance and templates. In Proceedings of the 7th European Conference of the Association for Computational Linguistics, pages 180-187.

Jonathan Ginzburg and Ivan Sag. 2000. English Interrogative Constructions. Studies in Constraint-based Lexicalism. CSLI Publications, Stanford, California.

Jonathan Ginzburg, Howard Gregory, and Shalom Lappin. 2001. SHARDS: Fragment resolution in dialogue. In Harry Bunt, Ielka van der Sluis, and Elias Thijse, editors, Proceedings of the 4 th International Workshop on Computational Semantics (IWCS-4), pages 156-172, Tilburg.
Jonathan Ginzburg. 2001. Clarification ellipsis and nominal anaphora. In H. Bunt, editor, Computing meaning, volume 2. Kluwer, Dordrecht.

Howard Gregory and Shalom Lappin. 1999. Antecedent contained ellipsis in HPSG. In G. Webelhuth, J. P. Koenig, and A. Kathol, editors, Lexical and Constructional Aspects of Linguistic Explanation, pages 331-356. CSLI Publications, Stanford.

Martin Kay. 1996. Chart generation. In Proceedings of the 34th Annual Meeting of the ACL, pages 200-204.

K. R. McKeown. 1985. Text Generation: Using Discourse Strategies and Focus Constraints to Generate Natural Language Text. Cambridge University Press, Cambridge.

Nicolas Nicolov and Chris Mellish. 2000. PROTECTOR: Efficient Generation with Lexicalized Grammars. In Recent Advances in Natural Language Processing, Current Issues in Linguistic Theory (CILT 189), pages 221-243. John Benjamin, Amsterdam \& Philadelphia.

Carl Pollard and Ivan Sag. 1994. Head Driven Phrase Structure Grammar. University of Chicago Press and CSLI Publications, Chicago.

Ehud Reiter. 1995. NLG vs. templates. In Proceedings of the Fifth European Workshop on Natural-Language Generation (ENLGW-1995), Leiden, The Netherlands.

Ivan Sag. 1997. English relative clause constructions. Journal of Linguistics, 33:431-484.

Stuart Shieber, Fernando Pereira, Gertjan van Noord, and Robert Moore. 1990. Semantichead-driven generation. Computational Linguistics, 16:30-42. 